\title{
Frequency and coding responses in verbal discrimination learning*
}

\author{
DOUGLAS L. MEDIN \\ The Rockefeller University, New' York, New York 10021
}

\begin{abstract}
Ss were given a verbal discrimination task where correct and incorrect stimuli appeared in one, two. or four different pairs in a within-Ss design. For a group tested with the usual anticipation procedure, correct repetitions helped and incorrect repetitions hurt performance. The pattern of results suggested that frequency is a salient cue in verbal discrimination learning but that coding or paired-associate processing may occur at the same time. Data from a second group of Ss for which frequency was a less salient cue gave more direct evidence for coding.
\end{abstract}

Since its initial formulation by Ekstrand, Wallace, and Underwood (1966), the frequency theory of verbal discrimination learning has received considerable favorable support. The basic idea is that frequency information provides the basis for discrimination. In the usual verbal discrimination task, pairs of words are presented, one word in each pair being arbitrarily selected as correct and the other as incorrect. In the anticipation method the pair is shown, a choice made, and then the correct word appears alone, so that the correct word appears twice as often as the incorrect word of a pair. Since frequency discrimination is quite accurate (e.g., Underwood, Zimmerman, \& Freund, 1971), it is plausible that frequency differences mediate verbal discrimination learning.

The most striking evidence in favor of this theory comes from an experiment by Underwood and Freund (1968), which varied the number of different pairs in which a correct or an incorrect word appeared. Intuitively speaking, repeating words can only help performance because if, for example, you learned that "DOG" is wrong in one pair, you would be able to use that information and avoid choosing "DOG" in other pairs. The theory predicts that increased repetitions of correct words should help learning but that repeating incorrect words might harm learning. because it would obscure frequency differences. The latter function would not be monotonic: performance should be worst with two repetitions of an incorrect word where there is no frequency differential and improve with larger numbers of repetitions of incorrect words. At first it may seem that the theory would predict that performance would be below chance if the frequency of wrong words was high enough, but the experiment varied frequency between Ss and the frequency theory assumes that Ss can either use the rule of choosing the most frequent or the least frequent words. Underwood and Freund observed that, as repetition of incorrect

*This research was supported by Grants GM16735 and MH16100 from the National Institute of General Medical Sciences. words increased, performance first decreased and then improved, as predicted.

The above results could not be predicted from a coding or paired-associate view of verbal discrimination that assumes correctness or incorrectness becomes linked by a code or stimulus-response bond to the stimuli, since repetitions could only help, according to these theories. However, there may be some peculiarities to the between-S paradigm used by Underwood and Freund (1969). In addition, Ss received an initial run through the list that might have allowed Ss to become familiar with the structure of the list before responding began.

The present study was similar to Underwood and Freund's, except that a within-S design was employed. Correct or incorrect stimuli could appear in one, two, or four different pairs. If responding is based solely on frequency discriminations, then it should be impossible to master the discrimination. because in some stimulus pairs the most frequent stimulus will be correct and in other pairs the most frequent stimulus will be incorrect. An anticipation method was used, but Ss were required to guess on the first trial. A second group of Ss was run in a condition in which feedback consisted of presentation of the item chosen, along with the associated outcome. For this group, frequency should be a much less salient cue, since the correct stimulus is not necessarily presented twice on each trial.

\section{METHOD}

Subjects

Forty college-age Ss were obtained in response to advertisements in a local newspaper (The Village Voice) and were paid $\$ 2$ each for an approximately 50 -min session. They were randomly assigned to one of the two groups.

\section{Apparatus}

An $8 \times 12 \mathrm{ft}$ experimental room contained an ASR-33 Teletype that was used for presentation of stimuli and outcomes. The Teletype was controlled by a PDP-8/I computer housed in an adjacent room. The computer was programmed to control stimulus presentation. response recording. outcome information. and intertrial intersals. The Ss responded by selecting a kerboard 
Table 1

Mean Errors Per Stimulus Pair for Correct and Incorrect Words Repeated in One. Two. or Four Different Pairs (Standard Deviations are in Parentheses)

\begin{tabular}{lccc}
\hline & \multicolumn{3}{c}{ Number of Repetitions } \\
\cline { 2 - 4 } & One & Two & Four \\
\hline $\begin{array}{l}\text { Anticipation Group } \\
\quad \text { Correct }\end{array}$ & 2.09 & 1.70 & 1.01 \\
$\quad$ Stimuli & $(1.11)$ & $(1.15)$ & $(0.78)$ \\
$\quad$ Incorrect & 2.09 & 2.94 & 2.96 \\
$\quad$ Stimuli & $(1.11)$ & $(0.81)$ & $(1.35)$ \\
Choice Group & & & \\
$\quad$ Correct & 3.26 & 2.85 & 1.99 \\
Stimuli & $(1.38)$ & $(1.36)$ & $(1.36)$ \\
Incorrect & 3.26 & 3.05 & 2.68 \\
Stimuli & $(1.38)$ & $(1.03)$ & $(1.13)$ \\
\hline
\end{tabular}

letter covered with an arrow pointing to the left (designating choice of the left stimulus) or an adjacent letter covered with an arrow pointing to the right.

Stimuli were 30 different CCCs of $21 \%-29 \%$ association value (Witmer, 1935). For each S. stimuli were paired randomly to reate 28 pairs. with the following constraints: Two stimuli were selected to appear in four different pairs as correct. two were selected to appear in two different pairs as correct. two appeared in four different stimuli as incorrect. and two appeared in two different stimuli as incorrect. For any repeated stimulus, the other member of a pair appeared just in one pair. Finally, the correct and incorrect stimuli appeared just once in four pairs. If an item was correct in one pair. it was correct in all other pairs it was part of: similarly. an item incorrect in one pair was always incorrect.

\section{Procedure}

Ss received nine runs through the 28-pair list of CCCs. The list order was determined randomly for each $S$ for each run. with the restriction that at least three trials intervened between any repetition of a pair or of any member of a pair. in the case of those stimuli appearing in more than one pair. For each trial, (1) the stimuli were typed out on the Teletype. (2) the $S$ chose either the left- or right-hand CCC, and (3) the Teletype printed the outcome. displaying it for $1 \mathrm{sec}$ before the next trial began.

Ss were given the following instructions. "On each trial you will see two words. The computer has assigned one point to one of the words and zero points to the other. Your task is to choose the word that has one point paired with it. The first time you see a pair of words. you'll just have to guess which one has one point paired with it. However. the words will be repeated and the assignment of points to words will not change, so that by remembering the previous trials, eventually you can always choose the correct word. If you know that a word had one point associated with it, you should always choose it, while, if the word has zero points paired with it, you should avoid choosing it." The 252-trial test session lasted 40-50 min.

The two groups differed only in the manner in which the results of a trial were presented. For the usual anticipation group. the trial always ended with the display of the correct stimulus along with " +1 ." For the choice group, the trial ended with the display of the S's choice along with a "+1" or a "+0." depending upon whether the S's choice was correct or incorrect. As a result of these procedures. for the anticipation group the correct member of a pair appeared twice as often as the incorrect stimulus. but for the choice group there was no built-in frequency cue. at least until performance on a pair was greater than chance.

\section{RESULTS AND DISCUSSION}

Table 1 gives the mean errors per pair for the various repetition conditions for the anticipation and choice groups. Correct repetitions facilitated performance in both groups, and the anticipation group made distinctly fewer errors than the choice group. Repetition of incorrect stimuli was associated with fewer errors for the choice group but in the anticipation group led to worse performance, contrary to a simple coding or paired-associate conception of verbal discrimination learning. Statistical analyses confirm these trends. An analysis of variance shows the effects of groups $(\mathrm{F}=5.21 . \mathrm{df}=1 / 38, \mathrm{p}<.05)$, repetition conditions $(F=14.10, \mathrm{df}=4 / 152, \mathrm{p}<.01)$, and their interaction $(F=4.50, \quad d f=4 / 152 . \quad p<.01)$ to be significant. Individual $F$ tests with $1 \mathrm{df}$ in the numerator show that the anticipation group excelled the choice group for the stimuli appearing in just one pair and for the conditions employing correct repetitions. Individual t tests showed that four repetitions was better than a single occurrence for both groups when correct stimuli were repeated and. for the choice group. when incorrect stimuli were repeated $(p<.05)$. In the anticipation group, four incorrect repetitions was significantly worse than a single occurrence.

Frequency played a major role in learning for the anticipation group. It was as if $\mathrm{Ss}$ chose the more frequent member of a pair regardless of its value, producing improvement for repeated correct and a decrement for repeated incorrect stimuli. But that is not the whole picture. If frequency were the only cue used, Ss would never have mastered the discrimination. Yet all but two Ss were correct on each of their last two runs through the list. Both coding and frequency selection could have occurred at the same time in the anticipation group. Repetitions only helped the choice group, yet their overall performance was significantly worse than that of the anticipation group. This would follow if choice Ss were less likely or less able to use frequency as a cue. A closer analysis of the data tends to support this idea.

If frequency accrues through rehearsal. Ss in the anticipation group may have been able to overcome the E-defined frequency effects for repeated incorrect stimuli by extra rehearsal of the correct words. Performance prior to the last error suggests that this was not the case. For the anticipation group, the probability of a correct response prior to the last error for four incorrect repetitions was .39 , for two incorrect repetitions was .46 , for one repetition was .56 , for two correct repetitions was .60 , and for four correct repetitions was .77 , based on $541,299.232,196$, and 316 responses, respectively. In other words. Ss were below chance for repeated incorrect stimuli and above chance for repeated correct stimuli prior to their last error on a pair. This suggests that these Ss were not gradually overcoming frequency differences to solve the 
discrimination. The comparable data for the choice group show a completely different pattern. Probability correct prior to the last error ranged from .51 to .54 for the various conditions, suggesting no special decrement or facilitation by repetition conditions before the last error. One cannot tell from these data whether Ss failed to try to use frequency or whether it simply was the case that frequency provided no useful information prior to the last error.

One could account for the present data by assuming that frequency and coding processes operate in parallel in verbal discrimination learning. Where frequency and correctness are perfectly correlated, both processes would work together, with frequency perhaps being dominant because it is such a salient feature. However, in situations contrived to produce both positive and negative correlations between frequency and correctness, the coding process may become more evident. For repeated incorrect stimuli in the usual anticipation procedure, it is as if probability correct would eventually have fallen to zero but for the coding process. On the other hand, where experimental conditions decrease the salience of frequency, as in the choice group, the data begin to appear as if a single all-or-none coding process were operating.
In summary, the present experiment shows that frequency is a major cue in verbal discrimination learning, even when not perfectly correlated with correctness. But the data also suggest that stimuli become associated with outcomes of correctness and incorrectness in the course of learning. Both processes appear to operate.

\section{REFERENCES}

Ekstrand, B. R., Wallace, W. P., \& Underwood, B. J. A frequency theory of verbal discrimination learning. Psychological Review, 1966, 73, 566-578.

Underwood, B. J., \& F reund, J. S. Verbal discrimination learning with varying numbers of right and wrong items. American Journal of Psychology, 1969, 82, 198-202.

Underwood. B. J., Zimmerman, J., \& F reund, J. S. Retention of frequency information with observations on recognition and recall. Journal of Experimental Psychology, 1971, 87, 149-162.

Witmer, L. R. The association value of three-place consonant syllables. Journal of Genetic Psychology, 1935, 47, 337-359.

(Received for publication March 21, 1973; revision received April 27, 1973.) 